\title{
Association of the Dietary-Based Diabetes-Risk Score (DDS) with the risk of gestational diabetes mellitus in the Seguimiento Universidad de Navarra (SUN) project
}

\author{
Mikel Donazar-Ezcurra ${ }^{1,2}$, Cristina Lopez-del Burgo ${ }^{1,3,4,5}$, Miguel A. Martinez-Gonzalez ${ }^{1,3,4,6}$, \\ Ligia J. Dominguez ${ }^{7}$, Francisco J. Basterra-Gortari ${ }^{1,4,8}$, Jokin de Irala ${ }^{1,3,4,5}$ and Maira Bes-Rastrollo ${ }^{1,3,4 * *}$ \\ ${ }^{1}$ Department of Preventive Medicine and Public Health, University of Navarra, Pamplona, Spain \\ ${ }^{2}$ Department Obstetrics and Gynaecology, Complejo Hospitalario de Navarra-Osasunbidea-IDISNA, Pamplona, Spain \\ ${ }^{3}$ CIBER Fisiopatologia de la Obesidad y Nutricion (CIBERobn), Instituto de Salud Carlos III, Madrid, Spain \\ ${ }^{4}$ Navarra's Health Research Institute (IDISNA), Pamplona, Spain \\ ${ }^{5}$ Institute for Culture and Society (ICS), University of Navarra, Pamplona, Spain \\ ${ }^{6}$ Department of Nutrition, Harvard TH Chan School of Public Health, Boston, MA, USA \\ ${ }^{7}$ Geriatric Unit - Department of Internal Medicine and Specialties, University of Palermo, Palermo, Italy \\ ${ }^{8}$ Department of Internal Medicine (Endocrinology), Hospital Reina Sofia, Osasunbidea-IDISNA, Tudela, Spain
}

(Submitted 6 December 2018 - Final revision received 4 June 2019 - Accepted 18 June 2019)

\section{Abstract}

It is crucial to identify people at risk for type 2 diabetes mellitus (T2DM) and gestational diabetes mellitus (GDM) to implement preventive interventions in order to address these pandemics. A simple score exclusively based on dietary components, the Dietary-Based DiabetesRisk Score (DDS) showed a strong inverse association with incident T2DM. The objective was to assess the association between DDS and the risk of GDM in a cohort of Spanish university graduates. The 'Seguimiento Universidad de Navarra' project is a prospective and dynamic cohort which included data of 3455 women who notified pregnancies between 1999 and 2012. The diagnosis of GDM is self-reported and further confirmed by physicians. A validated 136-item semi-quantitative FFQ was used to assess pre-gestational dietary habits. The development of the DDS was aimed to quantify the association between the adherence to this a priori dietary score and T2DM incidence. The score exclusively included dietary components (nine food groups with reported inverse associations with T2DM incidence and three food groups which reported direct associations with T2DM). Three categories of adherence to the DDS were assessed: low (11-24), intermediate (25-39) and high (40-60). The upper category showed an independent inverse association with the risk of incident GDM compared with the lowest category (multivariateadjusted OR 0.48; $95 \%$ CI 0.24, 0.99; $P$ for linear trend: 0.01). Several sensitivity analyses supported the robustness of these results. These results reinforce the importance of pre-gestational dietary habits for reducing GDM and provide a brief tool to practically assess the relevant dietary habits in clinical practice.

Key words: Diabetes dietary score: Gestational diabetes risk: Cohort studies: Diabetes prevention

Pregnancy produces insulin resistance in women mainly due to the placental secretion of diabetogenic hormones such as growth hormone and placental lactogen ${ }^{(1)}$. The objective of this metabolic change during pregnancy is to ensure the supply of glucose and nutrients to the fetus. Gestational diabetes mellitus (GDM) develops when the pancreatic function of the pregnant woman is not able to overcome this insulin resistance ${ }^{(2)}$, leading to a hyperglycaemic state in pregnant women.

Although the prevalence of GDM has historically been about 6-7 \%, actual data from the most important international scientific organisations considerably differ ${ }^{(1)}$, with estimates of the global prevalence of GDM from 1 to $50 \%$ (American Diabetes Association 2-19 \%; Carpenter and Coustan 3.6-38 \%; National Diabetes Data Group 1.4-50 \%; and WHO $2-24.5 \%)^{(3)}$. These different data are, in large part, the consequence of a non-universally standardised method for the screening and diagnosis of $\mathrm{GDM}^{(3)}$. However, there is enough evidence that GDM prevalence is increasing worldwide ${ }^{(4)}$. The most important reasons for this growing prevalence are the increasing maternal age and rates of obesity among women of reproductive

Abbreviation DDS, Dietary-Based Diabetes-Risk Score; GDM, gestational diabetes mellitus; SUN, Seguimiento Universidad de Navarra; T2DM, type 2 diabetes mellitus.

* Corresponding author: Maira Bes-Rastrollo, email mbes@unav.es 
age, and the higher proportion of the world population following a Western-type diet and lifestyles ${ }^{(4,5)}$

This is in line with the increasing prevalence of type 2 diabetes (T2DM). The estimates are that, by 2040, there will be 227 million more people worldwide with T2DM than in 2015, and many of these new diagnoses will be among young adults, thus also in women of reproductive age. Moreover, the main causes of the pandemic of T2DM are also those described above for $\mathrm{GDM}^{(6)}$

There is strong evidence showing that diet modifications are able to decrease the incidence of T2DM and that the effects of these dietary changes are likely to persist in the long term ${ }^{(7-9)}$. In contrast, there are few articles studying lifestyle and dietary habits using scores in the risk of $\mathrm{GDM}^{(10-14)}$. To the best of our knowledge, there is scarce evidence in the literature that dietary interventions can reduce the risk of $\mathrm{GDM}^{(15)}$. A recent systematic review of clinical trials and observational studies shows the need of better designed prospective and intervention studies, providing high-quality data in order to disseminate the best available interventions for the prevention of GDM in women of reproductive age ${ }^{(15)}$.

Therefore, it is crucial to identify people at risk for T2DM and GDM to implement preventive interventions in order to stop these pandemics. A simple score exclusively based on dietary components, the Dietary-Based Diabetes-Risk Score (DDS) was developed using previously reported associations in the literature $^{(9)}$ and when applied to the Seguimiento Universidad de Navarra (SUN) cohort showed a strong inverse association with incident $\mathrm{T}_{2} \mathrm{DM}^{(9)}$. Hence, the objective of the present analyses was to evaluate this DDS with the risk of GDM in the SUN project.

\section{Methods}

\section{Study population}

The SUN project began in 1999 and it is an ongoing, prospective and dynamic cohort. It was designed to investigate associations between lifestyle and dietary habits with many health outcomes. All the participants of this cohort are Spanish university graduates, because this is one of the inclusion criteria. In brief, a mailed questionnaire was sent to invite participants regarding dietary habits, lifestyles and health conditions. At baseline, once the participants accept to participate in the SUN project, they receive a detailed questionnaire by mail. The voluntary response to this baseline mailed questionnaire was considered as informed consent to participate in the study. After the initial assessment, data are updated with successive follow-up questionnaires (every 2 years). The study protocol was conducted accordingly with the Declaration of Helsinki and the Institutional Review Board of the University of Navarra approved the study. The design and methods of the SUN project have been previously described $^{(16,17)}$.

For these analyses, we have used the most updated database available in December 2015, finishing the follow-up of the current study at this date. Of 13777 women in the database in December 2015, we excluded 544 women who responded the baseline questionnaire after March 2013 in order to guarantee

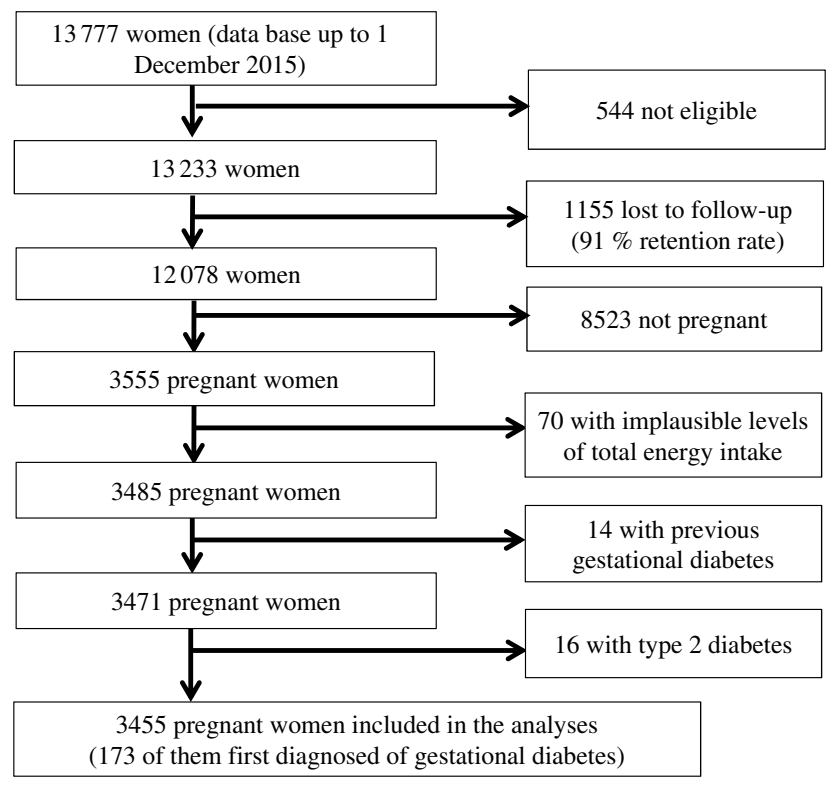

Fig. 1. Flow chart depicting the selection process among participants of the Seguimiento Universidad de Navarra (SUN) project to be included in the present analysis.

that analysed participants have been in the cohort enough time as to be able to respond at least the first follow-up questionnaire (2 years for the first follow-up questionnaire and 9 additional months to account for delays). We also excluded 1155 women who were lost during the follow-up. We also excluded from the analyses those women not reporting any pregnancy ( $n$ 8523), those out the predefined levels $(<1$ st percentile or $>$ 99th percentile) of total energy intake $(n 70)$ and those with a diagnosis of diabetes before the inception of the cohort ( $n$ 30). The final available population included 3455 women who reported at least one pregnancy during follow-up (Fig. 1).

\section{Dietary habits assessment}

A 136-food-item semi-quantitative FFQ assessed the dietary habits of the participants at the baseline questionnaire and after 10 years of follow-up. The FFQ asked for dietary intakes during the last year, and the frequency of consumption was: never, 1-3 times per month, once per week, 2-4 times per week, 5-6 times per week, once daily, 2-3 times daily, 4-6 times daily and 6 or more times daily. This FFQ has been repeatedly validated and described in detail ${ }^{(18,19)}$. The validity and reproducibility of this FFQ have been reported elsewhere ${ }^{(20,21)}$.

The dietary data of the questionnaires were entered to the database using optical reading machines and codifying open responses by nutritionists and dietitians of the SUN project.

A trained nutritionist and dietitian updated nutrient data bank with the latest available nutritional information included in Spanish food composition tables to take into account the dynamic feature of the cohort ${ }^{(22,23)}$.

Nutrient scores (nutrient derivations from the questionnaires using food composition tables) were computed with a computer 
software designed for this objective (12th version software of StataCorp).

In 2015, Dominguez et al. developed the DDS in the SUN project $^{(9)}$. This score is exclusively composed of dietary components which were obtained from published reports of previous cohorts showing their significant and consistent association with the risk of $\mathrm{T} 2 \mathrm{DM}^{(9)}$. The original aim by Dominguez et al. ${ }^{\left({ }^{(9)}\right.}$ was to quantify the association between adherence to the DDS and T2DM incidence. To create the DDS, they considered the consumption of nine food groups with an inverse association with T2DM incidence (vegetables, fruit, fibre, whole cereals, nuts, coffee, PUFA, low-fat dairy products and moderate alcohol consumption) and three food groups with a direct association with the incidence of T2DM (red meat, processed meat and sugarsweetened beverages) ${ }^{(24-26)}$. As the present analysis included women only (the T2DM analyses of Dominguez et al. ${ }^{(9)}$ included both men and women), we adjusted the consumption of each food for total energy intake using the residual method only for women $^{(27)}$, to minimise the potential measurement errors in dietary exposures (very likely non-differential) derived from the use of an FFQ to assess dietary habits. Then, the energyadjusted estimates for each food groups (residuals) were ranked in quintiles. For the T2DM risk-protective food groups, a value was established from 1 to 5 according to the consumption quintiles. In contrast, the ranking was reversed for the quintile consumption values of the three food groups which increased risk of T2DM (from 5 to 1 accordingly to the quintiles of consumption). Alcohol was valued in a different way, assigning five points for women who had moderate consumption (5-25 g/d) and zero points for others. Finally, assigned values of the twelve food groups consumption (nine protective and three increasing the risk) were summed in order to obtain the score; thereby, the final DDS could range from 11 to 60 points, the lowest and the highest adherence, respectively. Afterwards, three categories of adherence to the DDS were established: low (11-24), intermediate (25-39) and high (40-60). The establishment of these three categories was done by Dominguez et $a l{ }^{(9)}$ instead of doing quantiles in order to facilitate future similar comparative studies like this one and because these categories were more representative per se. Besides, it follows current epidemiological recommendations about how to categorise continuous variables ${ }^{(28)}$.

\section{Assessment of gestational diabetes mellitus}

The procedure for adjudication of GDM cases in the SUN project has been reported elsewhere ${ }^{(29)}$. In summary, women reporting at least one pregnancy and a new self-reported diagnosis of GDM in any follow-up questionnaire (sent every 2 years) were considered possible incident cases of GDM. At that point, an additional questionnaire was sent to those women requesting their medical reports. Furthermore, this additional questionnaire also inquired about previous glycaemic disorders, the diagnosis test results and the indicated treatment. With all these information, medical doctors of the SUN project confirmed or not each GDM diagnosis according to the responses to the questionnaires and the medical records mailed by the patients. In the present analyses, we only used confirmed GDM cases (20\% were not confirmed from the initial potential cases only based on selfreports).

As indicated above, there is not a universal 'gold standard' diagnosis procedure for GDM and different protocols are used in clinical habitual practice worldwide ${ }^{(30)}$. In Spain, the most common GDM diagnosis procedure is the one that follows a two-step approach during the 24-28 weeks of gestation; the first step is a $50-\mathrm{g}$ oral glucose challenge with a threshold of $140 \mathrm{mg} / \mathrm{dl}$ $(7 \cdot 8 \mathrm{mmol} / \mathrm{l})$. Those who screen positive undergo a diagnostic $3-\mathrm{h}$ 100-g oral glucose tolerance test with the cut-offs established in the Third Workshop-Conference on Gestational Diabetes Mellitus $^{(31,32)}$ : fasting plasma glucose $105 \mathrm{mg} / \mathrm{dl}(5.8 \mathrm{mmol} / \mathrm{l})$, 1-h value $190 \mathrm{mg} / \mathrm{dl}(10.6 \mathrm{mmol} / \mathrm{l}), 2$-h value $165 \mathrm{mg} / \mathrm{dl}$ (9.2 $\mathrm{mmol} / \mathrm{l})$ and 3 -h value $145 \mathrm{mg} / \mathrm{dl}(8.1 \mathrm{mmol} / \mathrm{l})$. These criteria were applied to the population of the SUN project.

\section{Non-dietary covariates}

Information on socio-demographic variables, anthropometric measurements (weight was measured before pregnancy), lifestyle habits (physical activity, smoking status) and other clinical covariates (parity, family history of diabetes, CVD, hypertension, chronic medication) was collected at baseline (before pregnancy). Self-reported anthropometric measurements (weight and BMI) have shown sufficient validity in a subsample of the SUN project ${ }^{(33)}$. Physical activity was measured before pregnancy with objective measurements in metabolic equivalent tasks (MET) per week using a previously validated questionnaire which has demonstrated an adequate correlation (Spearman coefficient of $0.51(P=0.002)$ ) in a subsample of this cohort ${ }^{(34)}$.

\section{Statistical analysis}

Only the information collected from the baseline FFQ (before pregnancy) was used to create the DDS. Proportions for categorical variables and means with standard deviations for continuous variables were calculated according to previously described categories of DDS adherence based on previously established cut-off values. In contrast to T2DM, the diagnosis of GDM depends on the fact of being pregnant, and therefore it does not depend on time. Therefore, for the present analysis, we used non-conditional logistic regression models estimating the OR with their $95 \% \mathrm{CI}$, taking women with the lowest adherence to the DDS (11-24 score points) as the reference category. After a crude analysis, we fitted a model adjusted for age and a multivariate-adjusted model. The multivariate model was adjusted for major non-dietary risk factors of GDM: age (years), BMI $\left(\mathrm{kg} / \mathrm{m}^{2}\right)$, presence of family history of diabetes (yes or no), smoking status (never/current/former), physical activity (MET $\mathrm{h} /$ week), parity (nulliparous/1-2 pregnancies/ $\geq 3$ pregnancies), multiple pregnancy (yes or no), hours of television watching (h/d), hours sitting down per d, CVD (yes or no) and hypertension prevalence (yes or no). We did not adjust our multivariate model for other dietary variables, such as total energy intake, because it may be in the causal mechanism linking the DDS with GDM. In fact, a higher value in the DDS score implied a lower total energy intake. The $P$-trend was calculated using likelihood ratio tests comparing the model without DDS and a model with a 
new variable with the median for each DDS category as a continuous one.

To account for dietary changes during follow-up, dietary data were updated after 10 years of follow-up for those participants with available information. To conduct repeated measures, generalised estimating equations models using binomial distribution and logit as the link function with an unstructured correlation matrix were used to assess the relationship between updated DDS (after 10 years of follow-up for those with available information) and the development of GDM. We adjusted for the same variables of the logistic models.

To assess the robustness of our results, we conducted several sensitivity analyses under different scenarios: (1) including only primiparous women, (2) excluding obese participants, (3) excluding women with multiple pregnancies, (4) excluding women with hypertension and/or CVD at baseline, (5) additionally adjusting for snacking between meals, and following a special diet, (6) changing cut-off values for total energy intake limits, (7) excluding women older than 40 years. We also conducted additional analyses classifying participants according to their quartiles of adherence to the DDS, considering those in the first quartile as the reference category. Similarly, we assessed the results for each additional point, and four-point, of adherence to the DDS.

The analyses were performed with the 12th version software of StataCorp. All tests were two-sided and statistical significance was set at cut-off of $P<0 \cdot 05$.

\section{Results}

\section{Baseline participants' characteristics}

The range of values for the DDS in the 3455 ever-pregnant women included in our analyses was from 15 to 55 points. According to previously established categories of DDS adherence (low (11-24), intermediate (25-39) and high (40-60)), dietary and non-dietary characteristics of the analysed pregnant women are shown in Table 1 . The intermediate category was the one with the highest number of participants ( $n 2531$ (73.3 \%)). Women with higher adherence to the DDS were on average older, more likely to be nulliparous and to have family history of diabetes, more physically active and exhibited with less frequency the habit of snacking between meals, while those with a higher total energy intake and higher consumption of fast food were more likely to belong to the lowest category of the DDS. As expected, the consumption of the nine nutritional factors assumed to be inversely associated with T2DM (except for the intake of PUFA) increased accordingly across increasing categories of the DDS. Conversely, the consumption of the three food groups assumed to be detrimental decreased across increasing categories of the DDS (Table 1).

The most notable differences across DDS adherence categories were for the consumption of vegetables, fruits, low-fat dairy products, whole-grain bread and nuts. Moreover, pregnant women in the highest category of adherence to DDS had greater intakes of carbohydrates, vitamins $\mathrm{C}$ and $\mathrm{D}$, folate, haeme Fe from haeme sources and fibre, whereas their intakes of total fat and total energy were lower.

\section{Longitudinal results}

Among the 3455 ever-pregnant women, 173 first diagnoses of GDM were identified during 35647 person-years of follow-up (mean follow-up: 10.4 years, range: 2-14 years), corresponding to an incidence of $5.01 \%$ between ever-pregnant women of the SUN project. GDM incidences for the low, intermediate and high categories of adherence to the DDS were 5.3, 5.5 and $3.2 \%$, respectively (Table 2 ).

When the association between GDM incidence and categories of pre-gestational DDS adherence was adjusted for potential nondietary confounders (age, BMI, family history of diabetes, smoking status, physical activity, parity, multiple pregnancy, hours of television watching, hours sitting down, CVD and hypertension prevalence), our finding was that the highest category of the DDS showed a lower risk of incident GDM compared with the lowest category (reference); multivariate-adjusted OR 0.48 (95\% CI 0.24, 0.99; $P$ for linear trend: 0.01) (Table 2). The crude and the age-adjusted model showed non-significant inverse trends (crude model OR 0.59 (95\% CI 0.29, 1.20; $P$ for linear trend: $0 \cdot 05)$ and age-adjusted OR 0.55 (95\% CI 0.27, 1.12; $P$ for linear trend: 0.03 ) for the high $v$. the low (reference) categories of adherence to the DDS), although the $P$ for trend for the age-adjusted model was statistically significant.

Updated pre-gestational DDS calculated with reported dietary data after 10 years of follow-up did not substantially change the reported association remaining the $P$ for trend statistically significant.

We conducted several sensitivity analyses in order to assess the robustness of our results (Table 3 ). In order to avoid possible confounding bias generated for experiences from previous pregnancies, we restricted the analysis to primiparous women. Including only primiparous pregnant women, the findings did not change when we compared the highest $v$. the lowest categories of adherence with the DDS (multivariate-adjusted model OR: 0.42; $95 \%$ CI 0.19, 0.92; $P$ for linear trend: 0.01). When we excluded obese participants and then compared the extreme categories of adherence to the DDS, the results did not change either (multivariate-adjusted model OR: $0 \cdot 45$; $95 \%$ CI $0 \cdot 22$, 0.94; $P$ for linear trend: 0.02). Moreover, the results did not change when we excluded women with multiple pregnancies (multivariate-adjusted model OR: 0.48; $95 \%$ CI 0.24, 0.99; $P$ for linear trend: $0 \cdot 01$ ). The results remained in the limit of significance when we excluded women older than 40 years of age (multivariate-adjusted model OR: 0.47; $95 \%$ CI 0.23, 1.00; $P$ for linear trend: $0 \cdot 01$ ). Although the point estimates were similar when we changed the exclusion criteria for extreme total energy intake, they lost their statistical significance (Table 3).

Those women in the highest quartile of adherence to the DDS presented a $45 \%$ lower odds of developing GDM (OR: 0.55; $95 \%$ CI $0.34,0.90 ; P$ for trend: $0 \cdot 015)$. Similarly, for each additional point of adherence in the DDS the odds decreased $3 \%$, and for each four points more of adherence to the DDS, changing from the lowest score to the highest score for each food category, the odds decreased $12 \%$, being these associations statistically significant in both cases (one-point increment: 0.97 (95\% CI $0.94,0.99)$, four-point increment: 0.88 (95\% CI 0.80, 0.97)). 
Table 1. Characteristics of 3455 pregnant women in the Seguimiento Universidad de Navarra (SUN) cohort according to categories of the Diabetes Dietary

Score (DDS) before pregnancy in the SUN project

(Numbers and percentages; mean values and standard deviations)

\begin{tabular}{|c|c|c|c|c|c|c|c|}
\hline & \multicolumn{6}{|c|}{ DDS } & \multirow[b]{3}{*}{$P$ for trend } \\
\hline & \multicolumn{2}{|c|}{ Low (11-24) } & \multicolumn{2}{|c|}{ Intermediate (25-39) } & \multicolumn{2}{|c|}{ High (40-60) } & \\
\hline & Mean & SD & Mean & SD & Mean & SD & \\
\hline \multicolumn{8}{|l|}{ Women } \\
\hline$n$ & & & & & & & \\
\hline$\%$ & & & & & & & \\
\hline Age (years) & $27 \cdot 6$ & 4.9 & $28 \cdot 3$ & 4.7 & 29.4 & 4.6 & $<0.001$ \\
\hline BMI $\left(\mathrm{kg} / \mathrm{m}^{2}\right)$ & $20 \cdot 9$ & $2 \cdot 6$ & 21.4 & $2 \cdot 6$ & 21.5 & $2 \cdot 6$ & 0.008 \\
\hline Family history of diabetes (\%) & \multicolumn{2}{|c|}{7.4} & \multicolumn{2}{|c|}{$10 \cdot 8$} & \multicolumn{2}{|c|}{$11 \cdot 8$} & 0.067 \\
\hline Smoking (\%) & & & & & & & 0.041 \\
\hline Current & \multicolumn{2}{|c|}{$26 \cdot 2$} & \multicolumn{2}{|c|}{$25 \cdot 0$} & \multicolumn{2}{|c|}{$24 \cdot 1$} & \\
\hline Former & \multicolumn{2}{|c|}{$16 \cdot 0$} & & & \multicolumn{2}{|c|}{$24 \cdot 1$} & \\
\hline Multiple pregnancy (\%) & \multirow{2}{*}{\multicolumn{2}{|c|}{$\begin{array}{c}0.4 \\
79.5\end{array}$}} & \multicolumn{2}{|c|}{0.2} & \multirow{2}{*}{\multicolumn{2}{|c|}{$\begin{array}{c}0.2 \\
83.5\end{array}$}} & 0.457 \\
\hline Primiparous (\%) & & & \multicolumn{2}{|c|}{81.6} & & & 0.136 \\
\hline Physical activity (MET-h/week) & $15 \cdot 0$ & $23 \cdot 8$ & $18 \cdot 0$ & $19 \cdot 1$ & $23 \cdot 3$ & $20 \cdot 6$ & 0.001 \\
\hline Television watching $(\mathrm{h} / \mathrm{d})$ & 1.8 & 1.2 & 1.8 & 1.3 & 1.6 & 1.3 & 0.092 \\
\hline Sitting down $(\mathrm{h} / \mathrm{d})$ & 5.6 & $2 \cdot 4$ & 5.5 & $2 \cdot 2$ & 5.4 & $2 \cdot 1$ & 0.404 \\
\hline Prevalence of hypertension (\%) & \multicolumn{2}{|c|}{1.6} & & & & & $0 \cdot 108$ \\
\hline Prevalence of CVD (\%) & & & & & & & 0.029 \\
\hline Food groups in the DDS & & & & & & & \\
\hline Vegetables (g/d) & 359.8 & $190 \cdot 7$ & $530 \cdot 7$ & $321 \cdot 7$ & 779.4 & $395 \cdot 2$ & $<0.001$ \\
\hline Fruit $(g / d)$ & 203.9 & $167 \cdot 8$ & 339.0 & 324.2 & $536 \cdot 2$ & 432.9 & $<0.001$ \\
\hline Fibre intake $(\mathrm{g} / \mathrm{d})$ & 21.9 & $7 \cdot 7$ & $27 \cdot 6$ & $12 \cdot 0$ & 37.9 & 14.6 & $<0.001$ \\
\hline Whole-grain bread $(\mathrm{g} / \mathrm{d})$ & 1.1 & $5 \cdot 0$ & $12 \cdot 2$ & 31.0 & 33.5 & $45 \cdot 6$ & $<0.001$ \\
\hline Nuts $(\mathrm{g} / \mathrm{d})$ & 3.8 & 5.9 & 5.4 & $9 \cdot 0$ & 11.8 & $17 \cdot 8$ & $<0.001$ \\
\hline Coffee (cups/d) & 0.7 & 1.0 & $1 \cdot 1$ & $1 \cdot 2$ & 1.5 & 1.3 & $<0.001$ \\
\hline Low-fat dairy products $(\mathrm{g} / \mathrm{d})^{*}$ & $132 \cdot 9$ & 218.9 & $251 \cdot 7$ & 247.4 & $372 \cdot 3$ & $256 \cdot 6$ & $<0.001$ \\
\hline Alcohol intake (g/d) & 1.8 & 3.8 & $2 \cdot 9$ & $4 \cdot 2$ & 4.7 & $5 \cdot 0$ & $<0.001$ \\
\hline Red meats (g/d) & $132 \cdot 6$ & $77 \cdot 4$ & $81 \cdot 0$ & $44 \cdot 0$ & $46 \cdot 8$ & $33 \cdot 6$ & $<0.001$ \\
\hline Processed meat products $(\mathrm{g} / \mathrm{d})$ & 83.4 & 61.4 & $51 \cdot 2$ & $35 \cdot 6$ & $34 \cdot 1$ & $28 \cdot 1$ & $<0.001$ \\
\hline Sugar-sweetened beverages $(\mathrm{ml} / \mathrm{d})$ & 84.4 & $116 \cdot 9$ & 48.4 & $80 \cdot 7$ & $24 \cdot 2$ & 47.5 & $<0.001$ \\
\hline Other food groups and dietary habits & & & & & & & \\
\hline Snacking (\%) & & & & & & & $<0.001$ \\
\hline Special diet $(\%) \dagger$ & & & & & & & $<0.001$ \\
\hline Fast food $(\mathrm{g} / \mathrm{d})$ & 34.0 & $22 \cdot 8$ & $25 \cdot 2$ & $21 \cdot 3$ & $18 \cdot 6$ & $16 \cdot 0$ & $<0.001$ \\
\hline Legumes (g/d) & $22 \cdot 2$ & $11 \cdot 8$ & $22 \cdot 2$ & $17 \cdot 6$ & $23 \cdot 6$ & $20 \cdot 1$ & 0.179 \\
\hline Cereals (g/d) & $121 \cdot 8$ & 84.4 & $98 \cdot 7$ & $69 \cdot 3$ & 105.4 & $77 \cdot 0$ & $<0.001$ \\
\hline Olive oil $(g / d)$ & $20 \cdot 7$ & $16 \cdot 5$ & 19.7 & 15.5 & $21 \cdot 8$ & $15 \cdot 4$ & 0.006 \\
\hline Eggs (g/d) & $26 \cdot 7$ & $13 \cdot 7$ & 23.4 & $16 \cdot 5$ & $19 \cdot 8$ & 11.5 & $<0.001$ \\
\hline Fish $(\mathrm{g} / \mathrm{d})$ & $96 \cdot 2$ & $95 \cdot 7$ & $95 \cdot 1$ & $68 \cdot 6$ & $115 \cdot 5$ & 69.9 & $<0.001$ \\
\hline Whole dairy products $(\mathrm{g} / \mathrm{d}) \ddagger$ & 385.5 & $276 \cdot 3$ & 217.9 & 203.7 & $126 \cdot 3$ & $139 \cdot 0$ & $<0.001$ \\
\hline Dietary intakes & & & & & & & \\
\hline Total energy $(\mathrm{kJ} / \mathrm{d})$ & $12386 \cdot 3$ & $3200 \cdot 3$ & $10400 \cdot 2$ & $3112 \cdot 1$ & 10111.5 & $1066 \cdot 9$ & $<0.001$ \\
\hline Carbohydrate ( $\%$ of energy) & 39.7 & 7.0 & 43.0 & 7.0 & $45 \cdot 7$ & 7.4 & $<0.001$ \\
\hline Protein (\% of energy) & $18 \cdot 1$ & 3.4 & $18 \cdot 0$ & $3 \cdot 1$ & $18 \cdot 2$ & $3 \cdot 2$ & 0.259 \\
\hline Total fat (\% of energy) & $41 \cdot 8$ & 5.9 & $38 \cdot 2$ & $6 \cdot 2$ & 34.6 & 6.6 & $<0.001$ \\
\hline MUFA (\% of energy) & $17 \cdot 3$ & $3 \cdot 1$ & $16 \cdot 2$ & $3 \cdot 6$ & $15 \cdot 1$ & 3.9 & $<0.001$ \\
\hline PUFA (\% of energy) & 5.5 & 1.9 & 5.4 & 1.6 & $5 \cdot 1$ & 1.6 & 0.001 \\
\hline SFA ( $\%$ of energy) & $15 \cdot 6$ & $3 \cdot 3$ & $13 \cdot 2$ & $2 \cdot 9$ & $10 \cdot 7$ & $2 \cdot 7$ & $<0.001$ \\
\hline Vitamin $C(\mathrm{mg} / \mathrm{d})$ & 211.9 & $105 \cdot 3$ & 288.9 & $155 \cdot 1$ & $405 \cdot 6$ & 179.8 & $<0.001$ \\
\hline Vitamin D $(\mu \mathrm{g} / \mathrm{d})$ & 3.9 & $3 \cdot 2$ & 3.7 & $2 \cdot 7$ & 4.6 & $3 \cdot 1$ & $<0.001$ \\
\hline Fe from haeme sources $(\mathrm{mg} / \mathrm{d})$ & $18 \cdot 1$ & $5 \cdot 4$ & $17 \cdot 4$ & $5 \cdot 7$ & $19 \cdot 7$ & $6 \cdot 4$ & $<0.001$ \\
\hline Folate $(\mu \mathrm{g} / \mathrm{d})$ & 338.7 & $129 \cdot 0$ & $415 \cdot 6$ & 173.3 & 556.5 & $202 \cdot 6$ & $<0.001$ \\
\hline
\end{tabular}

MET, metabolic equivalent tasks.

* Low-fat milk, non-fat milk, skimmed yogurt, fresh cheese (Burgos cheese, goat cheese).

† For example, hypoenergetic, low-Na, hypolipidaemic, fibre-rich diets.

¥ Whole milk, sweetened condensed milk, cream, milk shake, whole yogurt, Petit Suisse cheese, curd, cheese cream or cheese wedge, old cheese (hard and semi-hard cheese (Swiss/Emmental cheese, Manchego cheese, etc.), other cheese, custard, ice cream.

\section{Discussion}

The DDS proposed by Dominguez et al. in the SUN project ${ }^{(9)}$ to quantify the association between a composite dietary index and T2DM could be applied as a useful tool for the assessment of the dietary risk of GDM. This is an a priori score composed of several specific nutritional components with consistent inverse or direct associations with T2DM ${ }^{(24-26)}$. Since T2DM and GDM share the majority of risk factors besides having a very similar aetiopathogenesis, the rationale of our study was to evaluate the performance of this score for the prevention of GDM.

The results of these analyses found that a pre-pregnancy high adherence to the DDS decreases significantly the risk of 
Table 2. Risk of incident gestational diabetes mellitus (GDM) according to baseline categories of the Diabetes Dietary Score (DDS) and updated DDS after 10 years for the repeated measures in the Seguimiento Universidad de Navarra (SUN) project

(Odds ratios and $95 \%$ confidence intervals)

\begin{tabular}{|c|c|c|c|c|c|c|c|}
\hline & \multicolumn{6}{|c|}{ DDS } & \multirow[b]{3}{*}{$P$ for trend } \\
\hline & \multicolumn{2}{|c|}{ Low (11-24) } & \multicolumn{2}{|c|}{ Intermediate (25-39) } & \multicolumn{2}{|c|}{ High (40-60) } & \\
\hline & OR & $95 \% \mathrm{Cl}$ & OR & $95 \% \mathrm{Cl}$ & OR & $95 \% \mathrm{Cl}$ & \\
\hline$n$ & \multicolumn{2}{|c|}{244} & \multicolumn{2}{|c|}{2531} & \multicolumn{2}{|c|}{680} & \\
\hline \multicolumn{8}{|l|}{ Incident GDM } \\
\hline Number & \multirow{2}{*}{\multicolumn{2}{|c|}{$\begin{array}{c}13 \\
5 \cdot 3\end{array}$}} & \multirow{2}{*}{\multicolumn{2}{|c|}{$\begin{array}{l}138 \\
5.5\end{array}$}} & \multirow{2}{*}{\multicolumn{2}{|c|}{$\begin{array}{l}22 \\
3.2\end{array}$}} & 0.09 \\
\hline$\%$ & & & & & & & \\
\hline Crude & 1 & Ref. & 1.02 & $0.57,1.84$ & 0.59 & $0.29,1.20$ & 0.05 \\
\hline Age-adjusted model & 1 & Ref. & 0.99 & $0.55,1.79$ & 0.55 & $0.27,1.12$ & 0.03 \\
\hline Multivariate-adjusted model ${ }^{\star}$ & 1 & Ref. & 0.90 & $0.50,1.62$ & 0.48 & $0.24,0.99$ & 0.01 \\
\hline Multivariate-adjusted model ${ }^{*}$ repeated measures $\dagger$ & 1 & Ref. & 0.98 & $0.52,1.85$ & 0.53 & $0.24,1 \cdot 14$ & 0.01 \\
\hline
\end{tabular}

Table 3. Sensitivity analysis of adjusted ${ }^{*}$ risk for incident gestational diabetes mellitus (GDM) according to categories of adherence to the Diabetes Dietary Score (DDS) in the Seguimiento Universidad de Navarra (SUN) project 1999-2013

(Odds ratios and $95 \%$ confidence intervals)

\begin{tabular}{|c|c|c|c|c|c|c|c|c|c|}
\hline & \multirow{3}{*}{$\begin{array}{c}\text { Cases of } \\
\text { incident GDM }\end{array}$} & \multirow[b]{3}{*}{$n$} & \multicolumn{6}{|c|}{ DDS } & \multirow{3}{*}{$\begin{array}{l}P \text { for } \\
\text { trend }\end{array}$} \\
\hline & & & \multicolumn{2}{|c|}{ Low (11-24) } & \multicolumn{2}{|c|}{$\begin{array}{l}\text { Intermediate } \\
\quad(25-39)\end{array}$} & \multicolumn{2}{|c|}{ High (40-60) } & \\
\hline & & & OR & $95 \% \mathrm{Cl}$ & OR & $95 \% \mathrm{Cl}$ & OR & $95 \% \mathrm{Cl}$ & \\
\hline Overall sample* & 173 & 3455 & 1 & Ref. & 0.90 & $0.50,1.62$ & 0.48 & $0.24,0.99$ & 0.01 \\
\hline Including only primiparous women & 144 & 2827 & 1 & Ref. & 0.88 & $0.46,1.67$ & 0.42 & $0.19,0.92$ & 0.01 \\
\hline Excluding obese participants & 167 & 3414 & 1 & Ref. & 0.87 & $0.48,1.57$ & 0.45 & $0.22,0.94$ & 0.02 \\
\hline Excluding women with multiple pregnancies & 173 & 3448 & 1 & Ref. & 0.90 & $0.50,1.62$ & 0.48 & $0.24,0.99$ & 0.01 \\
\hline $\begin{array}{l}\text { Excluding participants with hypertension and/or } \\
\text { CVD at baseline }\end{array}$ & 169 & 3358 & & Ref. & 0.96 & $0.52,1.78$ & 0.54 & $0 \cdot 26,1 \cdot 12$ & 0.02 \\
\hline $\begin{array}{l}\text { Overall sample* additionally adjusted for snacking } \\
\text { and following a special diet }\end{array}$ & 173 & 3455 & 11 & Ref. & 0.90 & $0.50,1.63$ & 0.49 & $0.24,1.00$ & 0.02 \\
\hline Energy limits between $>2092$ and $<14644 \mathrm{~kJ} / \mathrm{d}$ & 147 & 3102 & 1 & Ref. & 0.87 & $0.44,1.69$ & 0.49 & $0.22,1.09$ & 0.09 \\
\hline Energy limits between fifth and 95th percentiles & 146 & 3111 & 1 & Ref. & 0.95 & $0.49,1.85$ & 0.61 & $0.28,1.33$ & 0.18 \\
\hline Excluding $>40$-year-old women & 171 & 3394 & 1 & Ref. & 0.94 & $0.51,1.74$ & 0.47 & $0.23,1.00$ & 0.01 \\
\hline
\end{tabular}

${ }^{*}$ Model adjusted for age, BMI, family history of diabetes, smoking status, physical activity, parity, multiple pregnancy, hours of television watching, hours sitting down, CVD and hypertension prevalence at baseline.

developing GDM when a woman became pregnant. Although the protective association was not as strong to prevent GDM as it was for T2DM (for T2DM multivariate-adjusted HR 0.32 (95\% CI 0.14, 0.69) for the high $v$. the low categories of adherence to the DDS, and multivariate-adjusted OR 0.48 (95\% CI $0 \cdot 24,0 \cdot 99)$ for GDM risk), the present results provide quality data to find the best intervention for the primary prevention of GDM, although we only found significant association between the high adherence to the DDS group and the reference group in the multivariate-adjusted model. Moreover, it can be useful not only to classify pregnant women with high risk for GDM according to their dietary habits, but also to reinforce education on healthy dietary and lifestyle habits to women of reproductive age.

Nowadays, several T2DM risk scores are available to estimate the probability of developing T2DM in the future for a specific person ${ }^{(35)}$. Nevertheless, to the best of our knowledge, none of them have been used to predict the risk of GDM. The Nurses' Health Study II cohort conducted in the USA has provided the majority of the current evidence between dietary habits and GDM risk. On the one hand, it was shown that pre-pregnant women who consumed more animal protein and haeme Fe (red meat), animal fat and fatty foods (such as high-fat processed meats and fast food), sugar-sweetened cola, potatoes and sweets had increased risk of $\mathrm{GDM}^{(11,36-41)}$. Some of these findings have been verified in other populations ${ }^{(13,42,43)}$. On the other hand, it was published the association between the higher consumption of some healthy foods (vegetables, healthy protein sources such as vegetables, nuts and legumes, whole grain foods) with a decreased incidence of $\mathrm{GDM}^{(11,36,38)}$. Furthermore, Karamanos et al. found an inverse association between women who followed a Mediterranean dietary pattern (which has similarities to the DDS) and GDM incidence $^{(44)}$. These findings are of major importance taking into account the current pandemic of diabetes, which it is probably caused, at least in a considerable part, by an unhealthy dietary pattern and lifestyle ${ }^{(45)}$.

The aetiopathology of T2DM and GDM is very similar. Both types of diabetes are characterised by a state of insulin resistance which cannot be overcome through a compensatory higher secretion of pancreatic insulin. On the one hand, T2DM mainly develops in people with more body fat that they can cope with 
over time ${ }^{(46)}$. On the other hand, GDM occurs when the pancreatic function of the pregnant woman is not able to overcome the sudden insulin resistance produced by the diabetogenic placental hormones ${ }^{(1,2,47)}$. This metabolic challenge occurred during pregnancy may expose a predisposition to glucose intolerance. Furthermore, follow-up and prevention of T2DM is recommended for women with $\mathrm{GDM}^{(48)}$. These facts, together with other adverse outcomes of GDM, call for efforts to investigate modifiable risk factors of GDM. Due to a lack of randomised trials for the primary prevention of GDM, prospective cohort studies, such as the SUN project, can provide a good approach to nutritional factors responsible for the current diabetes pandemic.

The potential limitations of the present study are: (1) voluntary completion of the FFQ, which may conduct to some degree of selection bias (it makes more difficult to find associations). Nonetheless, some self-reported variables (weight and BMI) have been validated in subsamples of this cohort ${ }^{(33)}$; (2) although a FFQ is probably the best available method to assess dietary habits of large cohorts ${ }^{(49)}$, followed for a long time, it could be susceptible to information bias. However, the FFQ used has been previously validated ${ }^{(19-21)}$; (3) the associations between the DDS and T2DM are stronger ${ }^{(9)}$ than in the present study for GDM; (4) Dietary habits were not assessed during pregnancy. Women are susceptible to change their dietary pattern after knowing their pregnant state. Nevertheless, previous studies suggested that such changes are food-specific and not specifically for their dietary pat$\operatorname{tern}^{(41,50)}$; in addition, when the analyses were updated with repeated measures at 10 years of follow-up the results were in the same direction; (5) Probably due to a lack of statistical power, some of the sensitivity analyses lost their statistical significance, although their point estimates were similar; (6) Due to the fact that the SUN project participants are all graduates (highly educated), restriction was applied to minimise confounding bias by education, disease, presumed access to health care and socioeconomic status. Thus, the generalisability of our findings should be understood through common biological mechanisms following biological plausibility instead of statistical representativeness. Nevertheless, in the strict sense of external validity, our results can be generalised only to highly educated women. More studies are required to test the applicability of our findings to women from other populations.

The strengths of the study include: (1) large sample of persons with high retention rate; (2) prospective and dynamic design; (3) prolonged follow-up; (4) ability to control lifestyle and demographic confounders; (5) the use of a repeatedly validated $\mathrm{FFQ}^{(19-21)}$

\section{Conclusions}

In conclusion, a score exclusively based on dietary factors and designed to assess the risk of T2DM have also showed preventive association with GDM. Our results reinforce the importance of pre-gestational dietary habits to reduce gestational diabetes incidence and consequently T2DM in the future. The DDS may be appropriate for clinical practice because the nutritional factors included can be gathered in primary care or using selfadministered tools. Moreover, it may well be an educational tool for self-assessment of diabetes risk.

\section{Acknowledgements}

The authors thank the participants and the members of the SUN project.

Financial support was received from the Spanish Government-Instituto de Salud Carlos III, and the European Regional Development Fund (RD 06/0045, CIBER-OBN, Grants PI10/02658, PI10/02293, PI13/00615, PI14/01668, PI14/ 01798, PI14/01764, PI17/01795, and G03/140), the Navarra Regional Government (45/2011, 122/2014) and the University of Navarra.

C. L. B., M. A. M-G., J. D. I., L. J. D. and M. B-R. conceived, designed and conducted research. M. D-E. and M. B. R. analysed data and wrote the paper. C. L. B., M. A. M-G., L. J. D., F. J. B-G. and J. D. I. contributed to the discussion and reviewed/edited the manuscript. M. B-R. had primary responsibility for final content. All authors read and approved the final manuscript.

None of the authors has any conflicts of interest to declare.

\section{References}

1. Dirar AM \& Doupis J (2017) Gestational diabetes from A to Z. World J Diabetes 15, 489-511.

2. American Diabetes Association (2004) Gestational diabetes mellitus. Diabetes Care 27, S88-S90.

3. Hartling L, Dryden DM, Guthrie A, et al. (2012) Screening and diagnosing gestational diabetes mellitus. Evid Rep Technol Assess (Full Rep) 210, 1-327.

4. Ferrara A (2007) Increasing prevalence of gestational diabetes mellitus: a public health perspective. Diabetes Care 30 , 141-146.

5. Solomon CG, Willett WC, Carey VJ, et al. (1997) A prospective study of pregravid determinants of gestational diabetes mellitus. JAMA 278, 1078-1083.

6. International Diabetes Federation (2015) IDF Diabetes Atlas, 7th ed. http://www.idf.org/diabetesatlas.

7. Knowler WC, Barrett-Connor E, Fowler SE, et al. (2002) Diabetes Prevention Program Research Group. Reduction in the incidence of type 2 diabetes with lifestyle intervention or metformin. $N$ Engl J Med 346, 393-403.

8. Knowler WC, Fowler SE, Hamman RF, et al. (2009) Diabetes Prevention Program Research Group. 10-year follow-up of diabetes incidence and weight loss in the Diabetes Prevention Program Outcomes Study. Lancet 374, 1677-1686.

9. Dominguez LJ, Bes-Rastrollo $\mathrm{M}$, Basterra-Gortari FJ, et al. (2015) Association of a dietary score with incident type 2 diabetes: The Dietary-Based Diabetes-Risk Score (DDS). PLOS ONE 10, e0141760.

10. Zhang C, Tobias DK, Chavarro JE, et al. (2014) Adherence to healthy lifestyle and risk of gestational diabetes mellitus: prospective cohort study. BMJ 30, 349.

11. Bao W, Bowers K, Tobias DK, et al. (2014) Prepregnancy lowcarbohydrate dietary pattern and risk of gestational diabetes mellitus: a prospective cohort study. Am J Clin Nutr 99, 1378-1384.

12. Donazar-Ezcurra M, Lopez-Del Burgo C, Martinez-Gonzalez MA, et al. (2017) Pre-pregnancy adherences to empirically derived dietary patterns and gestational diabetes risk in a Mediterranean cohort: the Seguimiento Universidad de Navarra (SUN) project. Br J Nutr 118, 715-721.

13. Marí-Sanchis A, Díaz-Jurado G, Basterra-Gortari FJ, et al. (2018) Association between pre-pregnancy consumption of meat, iron intake, and the risk of gestational diabetes: the SUN project. Eur J Nutr 57, 939-949. 
14. Donazar-Ezcurra M, Lopez-Del Burgo C, Martinez-Gonzalez MA, et al. (2018) Soft drink consumption and gestational diabetes risk in the SUN project. Clin Nutr 37, 638-645

15. Donazar-Ezcurra M, López-Del Burgo C \& Bes-Rastrollo M (2017) Primary prevention of gestational diabetes mellitus through nutritional factors: a systematic review. $B M C$ Pregnancy Childbirth 17, 30.

16. Martinez-Gonzalez MA, Sanchez-Villegas A, De Irala J, et al. (2002) Mediterranean diet and stroke: objectives and design of the SUN project. Seguimiento Universidad de Navarra. Nutr Neurosci 5, 65-73.

17. Segui-Gomez M, de la Fuente C, Vazquez Z, et al. (2006) Cohort profile: the 'Seguimiento Universidad de Navarra' (SUN) study. Int J Epidemiol 35, 1417-1422.

18. Martinez-Gonzalez MA, de la Fuente-Arrillaga C, NunezCordoba JM, et al. (2008) Adherence to Mediterranean diet and risk of developing diabetes: prospective cohort study. BMJ 336, 1348-1351.

19. Martin-Moreno JM, Boyle P, Gorgojo L, et al. (1993) Development and validation of a food frequency questionnaire in Spain. Int J Epidemiol 22, 512-519.

20. Fernandez-Ballart JD, Pinol JL, Zazpe I, et al. (2010) Relative validity of a semi-quantitative food-frequency questionnaire in an elderly Mediterranean population of Spain. Br J Nutr 103, 1808-1816.

21. De la Fuente-Arrillaga C, VazquezRuiz Z, Bes-Rastrollo M, et al. (2010) Reproducibility of an FFQ validated in Spain. Public Health Nutr 13, 1364-1372.

22. Moreiras O, Carbajal A, Cabrera L, et al. (2013) Tablas de composición de alimentos, spanish (Food Composition Tables), 16th ed. Madrid: Piramide.

23. Mataix Verdu J (2009) Tabla de composición de alimentos, spanish (Food Composition Tables), 5th ed. Granada: Universidad de Granada.

24. Ardisson Korat AV, Willett WC \& Hu FB (2014) Diet, lifestyle, and genetic risk factors for type 2 diabetes: a review from the Nurses' Health Study, Nurses' Health Study 2, and Health Professionals' Follow-Up Study. Curr Nutr Rep 3, 345-354.

25. O'Connor LM, Lentjes MA, Luben RN, et al. (2014) Dietary dairy product intake and incident type 2 diabetes: a prospective study using dietary data from a 7-day food diary. Diabetologia 57, 909-917.

26. Bhupathiraju SN, Pan A, Manson JE, et al. (2014) Changes in coffee intake and subsequent risk of type 2 diabetes: three large cohorts of US men and women. Diabetologia 57, 1346-1354.

27. Hu FB, Stampfer MJ, Rimm E, et al. (1999) Dietary fat and coronary heart disease: a comparison of approaches for adjusting for total energy intake and modeling repeated dietary measurements. Am J Epidemiol 149, 531-540.

28. Greenland S (2008) Analysis of polytomous exposures and outcomes. In Modern Epidemiology, 3rd ed., pp. 303-304 [KJ Rothman, S Greenland and TL Lash, editors]. Philadelphia, PA: Lippincott Williams and Wilkins.

29. Dominguez LJ, Martinez-Gonzalez MA, Basterra-Gortari FJ, et al. (2014) Fast food consumption and gestational diabetes incidence in the SUN project. PLOS ONE 9, e106627

30. Buckley BS, Harreiter J, Damm P, et al. (2012) Gestational diabetes mellitus in Europe: prevalence, current screening practice and barriers to screening. A review. Diabetic Med 29, 844-854.

31. GEDE (2006) Grupo Español de Diabetes y Embarazo. Guía asistencial de diabetes mellitus y embarazo (3a edición)
(Spanish Group of Diabetes and Pregnancy. Guideline for diabetes mellitus and pregnancy ( $3 \mathrm{rd}$ ed.)). Av en Diabetol 22, 73-87.

32. Metzger BE \& The Organizing Committee (1991) Summary and Recommendations of the Third International WorkshopConference on Gestational Diabetes Mellitus. Diabetes Care 40, 197-201.

33. Bes-Rastrollo M, Perez Valdivieso JR, Sanchez-Villegas A, et al. (2005) Validación del peso e índice de masa corporal autodeclarados de los participantes de una cohorte de graduados universitarios. (Validation of self-reported weight and body mass index of participants in a cohort of university graduates). Rev Esp Obes 3, 183-189.

34. Martinez-Gonzalez MA, Lopez-Fontana C, Varo JJ, et al. (2005) Validation of the Spanish version of the physical activity questionnaire used in the Nurses' Health Study and the Health Professionals' Follow-up Study. Public Health Nutr 8 , 920-927.

35. Noble D, Mathur R, Dent T, et al. (2011) Risk models and scores for type 2 diabetes: systematic review. BMJ 343, d7163.

36. Bao W, Bowers K, Tobias DK, et al. (2013) Prepregnancy dietary protein intake, major dietary protein sources, and the risk of gestational diabetes mellitus. Diabetes Care 36, 2001-2008.

37. Bao W, Tobias DK, Olsen SF, et al. (2014) Pre-pregnancy fried food consumption and the risk of gestational diabetes mellitus: a prospective cohort study. Diabetologia 57, 2485-2491.

38. Bao W, Tobias DK, Hu FB, et al. (2016) Pre-pregnancy potato consumption and risk of gestational diabetes mellitus: prospective cohort study. BMJ 352, h6898.

39. Bowers K, Yeung E, Williams M, et al. (2011) A prospective study of prepregnancy dietary iron intake and risk for gestational diabetes mellitus. Diabetes Care 34, 1557-1563.

40. Bowers K, Tobias DK, Yeung E, et al. (2012) A prospective study of prepregnancy dietary fat intake and risk of gestational diabetes. Am J Clin Nutr 95, 446-453.

41. Chen L, Hu FB, Yeung E, et al. (2009) Prospective study of pregravid sugar sweetened beverage consumption and the risk of gestational diabetes mellitus. Diabetes Care 32, 2236-2241.

42. Qiu C, Frederick IO, Zhang C, et al. (2011) Risk of gestational diabetes mellitus in relation to maternal egg and cholesterol intake. Am J Epidemiol 173, 649-658.

43. Qiu C, Zhang C, Gelaye B, et al. (2011) Gestational diabetes mellitus in relation to maternal dietary heme iron and non heme iron intake. Diabetes Care 34, 1564-1569.

44. Karamanos B, Thanopoulou A, Anastasiou E, et al. (2014) Relation of the Mediterranean diet with the incidence of gestational diabetes. Eur J Clin Nutr 68, 8-13.

45. Smyth S \& Heron A (2006) Diabetes and obesity: the twin epidemics. Nat Med 12, 75-80.

46. Taylor R (2013) Type 2 diabetes. Etiology and reversibility. Diabetes Care 36, 1047-1055.

47. Buchanan TA \& Xiang AH (2005) Gestational diabetes mellitus. J Clin Invest 115, 485-491.

48. ACOG (2013) Committee on Practice Bulletins-Obstetrics. (Practice Bulletin No. 137: Gestational diabetes mellitus. Obstet Gynecol 122, 406.

49. Willett W (1998) Nutritional Epidemiology, 2nd ed. New York: Oxford University Press.

50. Rifas-Shiman SL, Rich-Edwards JW, Willett WC, et al. (2006) Changes in dietary intake from the first to the second trimester of pregnancy. Paediatr Perinat Epidemiol 20, 35-42. 